\title{
Long Period Variables in the Magellanic Clouds and the Galaxy
}

\author{
S.M.G. Hughes \\ Palomar Observatory 105-24, California Institute of Technology, \\ Pasadena CA 91125, USA
}

\begin{abstract}
New results ( last two years) on mainly observational properties of Long Period Variables (LPVs) in the Magellanic Clouds and the Galaxy are reviewed. These properties include the effects of metallicity variations on their mass loss rates, the use of AGB LPVs to map the stellar distributions of the Galactic disk and bulge, and using detailed observations of nearby Miras to investigate their structure and to obtain new parallax distances, with implications for the pulsation mode of Miras.
\end{abstract}

\section{Introduction}

It is a tradition to remark on the supposed confusion about what to call these things. The term LPV is simply a general classification of any red star with a long period of pulsation ( $\gtrsim 80$ days). The color of the star isn't critical as a classification criterion, but since $P \propto R^{2} / M$ (for the fundamental mode), only red stars are cool enough (and hence large enough) to pulsate at such long periods. In terms of evolutionary status, LPVs have been mainly identified to either be members of the asymptotic giant branch (AGB, which are shell-burning (helium and/or hydrogen) stars, around a mostly degenerate CO core), or more massive red supergiants (RSG, which are massive enough to burn helium, carbon, oxygen etc in their cores). In the past, most studies of LPVs have been of the Miras, which are LPVs with large $(\Delta V>2.5 \mathrm{mag})$ amplitudes of pulsation, and hence are generally the easiest to detect (they also have a well-established $P L$ relation). LPVs with $\Delta V<2.5 \mathrm{mag}$ are termed semi-regular variables (SRVs), which can be a misnomer as their periods are often just as stable as the Miras' (which, it should be pointed out, are known to vary). Because Miras are almost certainly all members of the upper AGB (eg. Hughes \& Wood 1990), they have tended to become the most commonly studied AGB stars. But our knowledge of the AGB is now being extended through studies of non-Mira LPVs both at the end of the AGB (variable OH/IR stars; eg Wood et al 1992) and near the beginning (SRVs; eg Willems \& de Jong 1988 and Kerschbaum \& Hron 1992; Jura \& Kleinmann 1992b). Since a large proportion of the AGB variables are likely to be non-Miras - Hughes \& Wood (1990) found that up to half the likely AGB LPVs in the LMC are the so-called SRa type, while in the Galaxy, Jura \& Kleinmann (1992b) found that $\sim 40 \%$ of the Galactic AGB LPVs are SRVs (with $\sim 30 \%$ in higher overtones of pulsation) - and most, or even all, red giants are likely to be variable (eg Eggen 1992), an attempt will be made to refer to all LPVs by their evolutionary status (ie RSG LPVs, AGB LPVs, and one possible RGB LPV). 
This review is mainly of results that have appeared since the last variable star conference (Wood 1990b; Whitelock 1990), with a personal bias towards the observational aspect of LPVs in the Galaxy and the Magellanic Clouds. The $P L$ relations of both the AGB and RSG LPVs continue to be of great interest, and are being used to probe the distributions of AGB LPVs (and the stellar populations they represent) in both the Galaxy's disk (thick and thin), and bulge, and also to potentially derive distances to galaxies at least as far as Virgo. Closer to home, high resolution studies of the very nearest LPVs are establishing new absolute distances and revealing detailed morphology of their atmospheres, and providing possible evidence to suggest that Mira may not be in the fundamental mode of pulsation.

\section{The Magellanic Clouds}

Wood, Moore \& Hughes (1991) have revjewed work done on LPVs in the Magellanic Clouds prior to July 1990. Since then, the only new LPVs to have been reported are a sample of variable OH/IR stars in the LMC (Wood et al 1992), which are the most difficult to find, as they are in their final stages of high mass loss and so are hidden (optically) behind thick circumstellar envelopes. In terms of $\mathrm{AGB}$ evolution, the $\mathrm{OH} / \mathrm{IR}$ stars are important as they are the link between the upper AGB and the planetary nebulae. Wood et al (1992) searched for OH maser and strong IR emission in IRAS-selected candidates in both the LMC and SMC, finding 6 point sources with $\mathrm{OH}$, and 19 with strong IR, all in the LMC. Many were found to be variable in the IR, with periods ranging from 930 to 1390 days. The stellar wind velocities were only 0.6 of those found for comparable Galactic $\mathrm{OH} / \mathrm{IR}$ stars, which is consistent with the LMC's lower metallicity. Mass loss rates of $\sim 10^{-5} M_{\odot} \mathrm{yr}^{-1}$ were derived, based on the wind velocities and IRAS and $\mathrm{OH}$ maser fluxes. Evidence for a possible metallicity-mass loss relation (for O-rich AGB LPVs) was found in the existence of two optically visible LPVs in the SMC with $P>1000$ days and $\mathrm{M}_{\mathrm{bol}}<-6$, which show no sign of large mass loss (ie no IRAS flux), in contrast to AGB LPVs of similar periods in the LMC and the Galaxy. Such a relation would imply that low metallicity stars would end up with higher white dwarf masses, and more will explode as supernovae. However, there is at least one SMC LPV, with a period of 800 days, that does have a dusty shell and therefore a high mass loss rate, but its luminosity is consistent with it being a carbon star. Therefore, it may be that low metallicity AGB stars can in fact have high mass loss rates if they turn into $\mathrm{C}$ stars. The Wood et al IRAS sources, when plotted on the same $\left(\mathrm{M}_{\mathrm{bol}}, \log P\right)$ plane as the other AGB LPVs in the LMC (Figure 1a), clearly shows all but one of them to be an extension of the optical AGB LPV sequence.

The non-nuclear-burning core of the AGB stars means that there exists a direct relation between their luminosities and their core masses, so that there is a natural upper luminosity limit, at $\mathrm{M}_{\mathrm{bol}} \sim-7.1$ (corresponding to the Chandrasekhar limiting mass for degenerate matter of $M \sim 1.4 M_{\odot}$ ). The inexactness of this luminosity limit is mainly due to uncertainties about mass loss rates, and while new models by Boothroyd \& Sackmann (1992), which include more realistic molecular opacities and the effects of any hot bottom burning, indicate the core mass-luminosity relation may not be applicable at high mass (which may explain the group of higher luminosity $M$ stars at $\log P \sim 2.8$ ), their model luminosities still do not go beyond the classic AGB luminosity limit. In contrast, the 
models by Blöcker \& Schönberner (1991), which also incorporate hot bottom burning, predict AGB luminosities well above this limit. However, this is not supported by the observations of Wood et al (Figure 1a) who find that the only OH/IR star above the AGB luminosity limit is a probable member of the LMC cluster NGC 1984, which would imply it has a mass of 15-20 $M_{\odot}$, and is therefore a supergiant (which is also consistent with its $K$ amplitude, which is very low for an $\mathrm{OH} / \mathrm{IR}$ variable, at just $0.2 \mathrm{mag}$ ).

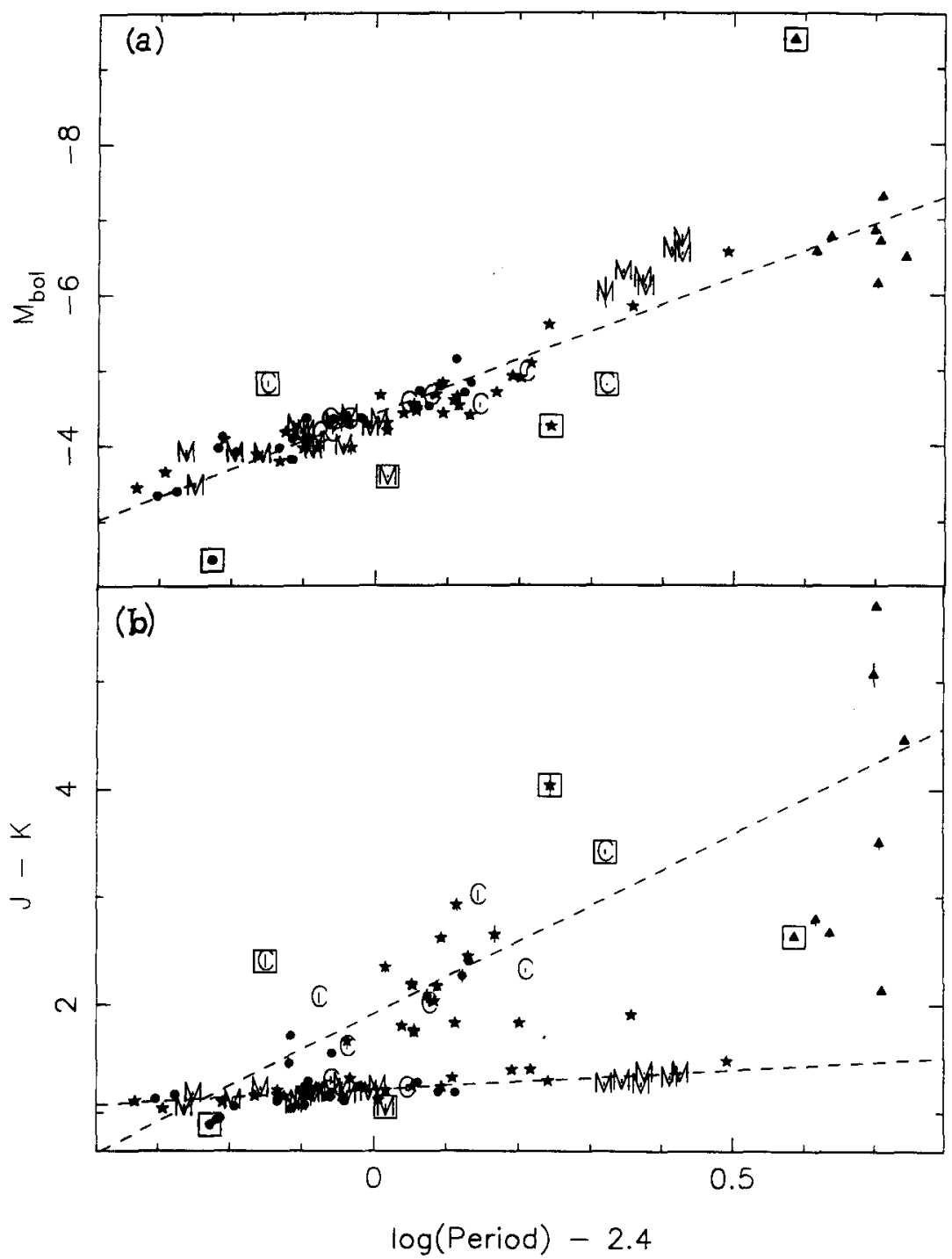

Figure $1 \mathrm{M}_{\mathrm{bol}}$ and $J-K$ vs $\log P$ diagrams for $102 \mathrm{AGB}$ LPVs (and one RSG LPV) in the LMC. The symbols have the following meaning: Objects with spectral types indicated by $\mathrm{M}$ or C, otherwise stars are from Glass et al (1990), triangles are OH/IR stars from Wood et al (1992) and circles are from Wood \& Hughes (1992). Outliers not used in the fits are within boxes. Error bars are the standard error on the mean. 
The known optical AGB LPVs have continued to be observed by Wood \& Hughes (1992), who have been monitoring 60 of the Hughes (1989) LPVs in the LMC, obtaining multi-epoch $J, K$ photometry, with $48 \mathrm{LPVs}$ now having 5 or more epochs. Combining these data with the Glass et al (1990) data used in a similar study by Feast et al (1989), the least squares solution to the $(K, \log P) P L$ relation, for $P<420$ days, is

$K=11.10( \pm 0.02)-3.75( \pm 0.14)[\log P-2.4], \quad \sigma=0.13, \quad$ for $N=79$.

Because most of the flux of these optical AGB LPVs is emitted in $K$, it is not surprising that the slope of this relation is not all that different from that obtained from the bolometric $P L$ relation, which for all AGB LPVs, including the Wood et al (1992) OH/IR variables is found to be

$$
\mathrm{M}_{\text {bol }}=-4.42( \pm 0.02)-3.61( \pm 0.18)[\log P-2.4], \quad \sigma=0.27, \quad \text { for } N=96 \text {, }
$$

where the indicated outliers in Figure 1a are excluded.

The 'faint' outliers in Figure 1a may possibly be semi-obscured stars with high mass loss rates, although a signature of high mass loss is a large $K$ amplitude (Whitelock 1990; Whitelock et al 1991), and the two with the shortest periods have low $K$ amplitudes ( $\sim 0.1 \mathrm{mag})$. One alternative, that they are background objects is unlikely, for the $\mathrm{M}$ star at least, as it has a measured radial velocity (Hughes et al 1991) consistent with LMC membership. Another possibility is that the shorter period objects are RGB stars.

The one 'bright' AGB outlier in Figure 1 is a carbon star, also with an LMC radial velocity. It is one of the shortest period $\mathrm{C}$ stars, and may well be in a higher overtone of pulsation, given that its amplitude ( $\Delta K=0.3 \mathrm{mag}$ ) is also low (Wood \& Bessell 1985). If it were in the first overtone, then its period of 178 days would imply a fundamental at $\sim 356$, not too different from a period of 363 predicted by the $P L$ relation from its $K$ mag. Alternatively, as the amplitude is so low, its also possible it has a poorly determined period.

The $(J-K, \log P) P C$ relations (Figure $1 \mathrm{~b}$ ), derived from those LPVs with measured spectral types, are

$J-K=1.215( \pm 0.014)+0.37( \pm 0.05)[\log P-2.4], \quad \sigma=0.06$, for $21 \mathrm{M}$ stars, and

$J-K=1.9( \pm 0.9)+3.3( \pm 7.3)[\log P-2.4], \quad \sigma=0.58, \quad$ for $10 \mathrm{C}$ stars.

The low dispersion in the $P C$ relation for the $\mathrm{M}$ stars is evidence that they obey a $P L C$ relation (see Feast et al 1989), but the divergent $P C$ relation for the $\mathrm{C}$ stars indicates this isn't generally applicable to all AGB LPVs.

The three $\mathrm{C}$ stars that lie on the $\mathrm{M}$ star $P C$ sequence are an anomaly, as their $K$ amplitudes of pulsation are all moderately high (at $\sim 0.6 \mathrm{mag}$ ), indicating their mass loss loss rates are $\sim 10^{-6} M_{\odot} \mathrm{yr}^{-1}$ (Whitelock 1990). Hence they are likely to be moderately evolved $\mathrm{C}$ stars, and should therefore have redder $J-K$ colors.

\section{The Galaxy}

\subsection{The Bulge LPVs}

Whitelock et al (1991) have obtained multiple epoch $J H K L$ photometry of a sample of IRAS-identified variable stars in the Galactic bulge. They find that the majority have the IR colors and periods of Mira variables, with very few having $P>560$. By estimating absolute luminosities from phase-lag and kinematic distances, they derived a bolometric $P L$ relation with a slope of -2.55 . Although this is much shallower than for the LMC, a large part of the discrepancy is probably due to the uncertain distances. The [12] $-[25] \mu \mathrm{m}$ 
IRAS colors (indicative of dust, and hence mass loss) were found to be linked to the pulsation amplitude in $K$. The density distribution was compared to flattened ellipsoidal models (with $a / b=1$ ), with a best fit being found for $c / a \sim 0.7$ and $\rho \propto a^{-3.2}$.

In a follow-up analysis, Whitelock \& Catchpole (1992) re-modelled the density distribution by a prolate ellipsoid. Their best fit indicates the bulge is likely to be a bar tilted at $\sim 45^{\circ}$ to the line of sight, in agreement with other recent studies, such as those by Nakada et al (1991) and Weinberg (1992a, 1992b).

\subsection{Disk LPVs}

Two major studies of the local AGB LPV density distributions have been made by Jura \& Kleinmann $(1992 \mathrm{a}, 1992 b)$ and Kershbaum \& Hron (1992), using the $P L$ relation to derive distances. Jura \& Kleinmann divided their O-rich Miras and semi-regulars (SRVs) into short $(P<300)$ and intermediate $(300<P<400)$ period groups. They found that the SRVs with $200<P<300$ have a similar distribution to the short period Miras, that of a 'thick' disk (scale height $\sim 550 \mathrm{pc}$ ), while the SRVs with $300<P<400$ and $100<P<150$ have a similar distribution to the intermediate period Miras, that of a 'thin' disk (scale height $\sim 240 \mathrm{pc}$ ). This would imply that most of the SRVs are also AGB stars, different from the Miras only in their pulsation amplitude, and probably being on the lower AGB. It also implies that the $100<P<150 \mathrm{SRVs}$ are of similar mass to the intermediate Miras, but in higher overtones of pulsation (which may explain their sometimes irregular periods).

Kershbaum \& Hron found essentially the same results, but derived a thicker 'thick' disk (scale height $\sim 780 \mathrm{pc}$ ) for the short period Miras. They also found evidence, from $V-[12 \mu \mathrm{m}]$ colors, for the $P<150 \mathrm{SRVs}$ to be 'hot' $\left(T_{\text {eff }}>3200 \mathrm{~K}\right)$.

Jura \& Kleinmann (1992a) estimated the number density for the 'thin' disk O-rich Miras to be $\sim 210 \mathrm{kpc}^{-3}$. Mass loss rates of $\sim 10^{-7} M_{\odot} \mathrm{yr}^{-1}$ were derived for both groups of Miras from their IRAS $60 \mu \mathrm{m}$ flux. Mass loss rates for the SRVs were estimated as $10^{-8}-10^{-7} M_{\odot} \mathrm{yr}^{-1}$.

Hron (1991) has investigated a potentially important relation between the metallicity of the short period $(P<200)$ Miras and their $\left(V_{\max }-K\right)_{0}$ color, combining a method used by Bessell et al (1986) with the theoretical formulae of Wood (1990a) concerning the relationship between an LPV's luminosity, temperature, mass and metallicity. Although this method is unable to measure an absolute metallicity for any individual Mira, and is limited to LPVs hotter than $3200 \mathrm{~K}$, nevertheless these preliminary results indicate a metallicity difference exists between the AGB LPVs with rotation velocities similar to the thin disk, and those AGB LPVs with a much lower rotation component and a velocity dispersion similar to that of the metal-rich RR Lyraes.

\subsection{Nearby LPVs}

High resolution imaging with HST, the VLA and mm telescopes, and now increasingly optical imaging from the ground (using both interferometric and aperture synthesis techniques) have resulted in detailed observations of the angular sizes, shapes and even detailed morphology of nearby Miras. Without being exhaustive, the following is a brief summary of papers that have appeared in the last two years concerning $R$ Aquarii and $o$ Ceti. 
Paresce et al.(1991), Hege et al.(1991), Hollis et al.(1992), Burgarella \& Paresce (1992), Solf (1992) and Lehto \& Johnson (1992) have all made detailed studies of R Aquarii, a 387 day Mira in a symbiotic system, producing images of a jet, a possible Herbig-Haro object, and providing evidence for two expanding shells.

Planesas et al (1990a, 1990b) mapped the CO radio emission of $o$ Ceti, finding a circumstellar envelope expansion velocity of only $3 \mathrm{~km} \mathrm{~s}^{-1}$, implying a mass loss rate of $\sim 1 \times 10^{-7} M_{\odot} \mathrm{yr}^{-1}$, evidence for bipolar gas outflow, and detecting both $\mathrm{SiO}$ and $\mathrm{CO}$ emission in the inner regions. Karovska et al (1991) and Haniff et al (1992) obtained optical images of the photosphere and molecular atmosphere of $o$ Ceti. Karovska et al measured departures from circular symmetry (at wavelengths 530 to $850 \mathrm{~nm}$ ). The direction of the assymmetry was found to change with phase, implying that it is related neither to the presence of the companion nor to any rotation of $o$ Ceti itself. Haniff et al found the sizes $o$ Ceti's elongated photosphere (best fitted by a uniformly illuminated disk with $a=55$ mas and $b / a=0.82$, at $700.7 \mathrm{~nm}$ and phase 0.3 ) and molecular atmosphere to be consistent with the model atmospheres of Bessell et al (1989), and confirmed the departures from circular symmetry, which they postulated to be due to the presence of a non-radial pulsation mode. Ridgway et al (1992) measured the angular diameter of $o$ Ceti to be 36 mas (best fit of a uniform disk, at phase 0.23 to 0.36). Fitting a limb darkened model gave a diameter of 43 mas, at an effective wavelength of $2.28 \mu \mathrm{m}$ (equivalent to $\sim 60$ mas at $700 \mathrm{~nm}$ ), implying an effective temperature of $2270 \mathrm{~K}$, consistent with IR colors, but less than the molecular excitation temperature (which would predict a diameter $\sim$ half that observed).

Two years ago it seemed that the question as to which mode of pulsation the Miras were in (fundamental or first overtone) had been resolved, with Wood (1990a, 1990b) agreeing with Willson (1982) that the pulsation models for first overtone could not reproduce the observed pulsation velocities, and therefore they had to be fundamental pulsators. In contrast, however, Tuchman (1991) has modelled the pulsation acceleration, and finds the observations are inconsistent with fundamental pulsators, but are in the first overtone. A part from velocity data, the other method of observationally determining the pulsation mode is to measure the pulsation constant $Q=P\left(M / R^{3}\right)^{1 / 2}$, where the period $P$ is easily measured, the mass $M$ is reasonably well constrained to be $\sim 1 M_{\odot}$, and the radius $R$ is inferred from the luminosity and effective temperature (see eg Wood 1992b). The weakest part of this measure thus lies in the determination of the luminosity and effective temperature. But high resolution studies have the potential to measure $R$ directly (once a reliable model for the effects of limb darkening is established). In the case of $o$ Ceti ( $P=$ 332), for example, the above diameter of 43 mas, at a parallax distance of $77 \mathrm{pc}$ (Jenkins 1952), gives a radius of $364 \pm 17 R_{\odot}$. Taking its mass as $1 M_{\odot}$, then $Q=0.048 \pm 0.003$, which the $P M R$ relation of Fox \& Wood (1982) would clearly identify as being in the first overtone. Even if the mass of $o$ Ceti was as uncertain as $1.6 \pm 1 M_{\odot}$ (Tuchman 1991), the resultant range in $Q(0.06 \pm 0.02)$ still favours the first overtone. However, if the radius predicted by the $\mathrm{CO}$ molecular excitation temperature is adopted, then $o$ Ceti would very definitely be in the fundamental mode.

As imaging instrumentation using adaptive optics become available, this field is destined to expand rapidly over the next few years.

At the same time as advances are being made in high resolution imaging, the next few 
years should also see a vast improvement in the number of Miras with parallax distances. For example, Gatewood (1992) has determined a new parallax for R Leo $(P=310)$, using the Multichannel Astrometric Photometer (MAP) at the Allegheny Observatory, giving a direct trigonometric distance to $\mathrm{R}$ Leo of $120 \pm 15 \mathrm{pc}$. This is remarkably similar to a $P L$ distance of $117 \mathrm{pc}$, derived by assuming the LMC $K, \log P$ relation with an LMC distance modulus of 18.5 , and taking $K=-2.4$ (Robertson \& Feast 1981). Such a result disagrees with theoretical calculations by Wood (1990a; 1990b), which predict that Galactic Miras should be about 0.4 mag fainter than an LMC Mira of the same period, due to their presumed metallicity difference.

However, a similar calculation for $o$ Ceti, with a (photographic) parallax distance of 77 pc (Jenkins 1952), and $K=-2.5$ (Robertson \& Feast 1981), implies a $P L$ distance of $\sim 120 \mathrm{pc}$. Gatewood is currently making MAP observations of $o$ Ceti, and it will be interesting to see if the distance remains the same.

HIPPARCOS is monitoring 245 large amplitude pulsators (Mennessier et al 1992), and it might be hoped there will soon exist a reasonably large sample of absolute Mira distances, which should help to resolve these issues.

\section{The Future}

Surveys for LPVs in the Magellanic Clouds are being conducted by Moore (1991) in the SMC, and Hughes \& Wood (ongoing) have initiated a new UK Schmidt multi-epoch plate survey of the eastern and western edges of the LMC, which should be complete in $\sim 1$ year. Another UK Schmidt LPV survey is also being conducted in 5 Galactic regions, 4 of which are in the bulge (Hughes \& Whitelock, ongoing).

The results of using HST wide field camera (WFC) exposures to successfully search for Cepheids in M81 (Hughes 1992; Stetson et al 1992, in preparation) and in IC4182 (Sandage et al 1992), proves that HST, even in its present handicapped state, could be used to search for AGB LPVs in external galaxies out to a distance modulus of 27.5 $(3.2 \mathrm{Mpc})$, and with WFPC2 out to $29.5(7.9 \mathrm{Mpc})$, providing the potential for studying relationships between galactic environment and AGB and AGB LPV evolution, and for tracing the stellar populations which the AGB LPVs represent in these galaxies.

Wood \& Bessell (1985) drew attention to the potential of a $P L$ relation for RSG LPVs as a means of measuring distances to galaxies. Recent CFHT observations by Pierce et al (1992a, 1992b, these proceedings) indicate that RSG LPVs can be detected in galaxies as distant as the Virgo cluster. RSG LPVs have the potential to become very useful distance indicators, as they are not only brighter than Cepheids (the brightest Cepheid has an $\mathrm{M}_{\text {bol }} \sim-6$, compared to $\mathrm{M}_{\text {bol }} \sim-9$ for RSG LPVs in the LMC), but also emit mostly in the red where uncertainties about internal extinction will be less.

\section{Acknowledgements}

My thanks to Peter Wood for all the help over the years, and to Patricia Whitelock and Michael Jura for helpful (e-mail) discussions and providing copies of preprints.

\section{References:}

Bessell M.S., Freeman K.C., Wood P.R. 1986 ApJ 310, 710.

Bessell M.S., Brett J.M., Scholz M., Wood P.R. 1989 A\&A 213, 209. 
Blöcker T., Schönberner D. 1991 A\&A 244, L43.

Boothroyd A.I., Sackmann I.-J. 1992, ApJ 393, L21.

Burgarella D., Paresce F., 1992 ApJ 389, L29.

Eggen O.J. 1992 AJ 104, 275.

Feast M.W. 1963 MNRAS 125, 367.

Feast M.W, Glass I.S., Whitelock P.A., Catchpole R. 1989 MNRAS 241, 375.

Fox M.W., Wood P.R. 1982 ApJ 259, 198.

Gatewood G. 1992 PASP 104, 23.

Glass I.S. 1985 Irish AJ 17, 1

Glass I.S., Whitelock P.A., Catchpole R., Feast M.W, Laney C.D. 1990 SAAO Circ. no.14, p 63.

Haniff C.A., Ghez A.M., Gorham P.W., Kulkarni S.R., Matthews K., Neugebauer G. 1992 AJ $103,1662$.

Hege E.K., Allen C.K., Cocke W.J. 1991 ApJ 381, 543.

Hollis J.M., Dorband J.E., Yusef-Zadeh F. 1992 ApJ 386, 293.

Hron J. 1991 A\&A 252, 583.

Hughes S.M.G. 1989 AJ 97, 1634.

Hughes S.M.G. 1992 in IAU Coll. 136: Stellar Photometry, Dublin August 1992, in press.

Hughes S.M.G., Wood P.R. 1990 AJ 99, 784.

Hughes S.M.G., Wood P.R., Reid N. 1991 AJ 101, 1304.

Jenkins L.F. 1952 General Catalogue of Trig. Stellar Parallaxes Yale University Press.

Jura M., Kleinmann S.G. 1992a ApJSupp 79, 105.

Jura M., Kleinmann S.G. 1992b ApJSupp in press.

Karovska M., Nisenson P., Papaliolios C., Boyle R.P. 1991 ApJ 374, L51.

Kerschbaum F., Hron J. 1992 A\&A, submitted.

Lehto H.J., Johnson D.R.H. 1992 Nature 355, 705.

Mennessier M.O., Barthés D., Boughaleb H., Figueras F., Mattei J.A. 1992 A\&A 258, 99.

Moore G.K.G. 1991 in IAU Symp. 148. The Magellanic Clouds, eds. R. Haynes and D. Milne (Kluwer: Dordrecht), p355.

Nakada Y., et al. 1991 Nature 353, 140.

Paresce F., et al 1991 ApJ 369, L67.

Pierce M.J., Welch D.L., McClure R.D., van den Bergh S., Racine R. 1992a these proceedings.

Pierce M.J., Crabtree D. $1992 \mathrm{~b}$ these proceedings.

Planesas P., Bachiller R., Martín-Pintado J., Bujarrabal V. 1990a ApJ 351, 263.

Planesas P., Kenney J.D.P., Bachiller R. 1990b ApJ 364, L9.

Ridgway S.T., Benson J.A., Dyck H.M., Townsley L.K., Hermann R.A. 1992 AJ submitted, NOAO preprint \# 418.

Robertson J.W. 1974 ApJ 191, 67.

Robertson B.S.C., Feast M.W. 1981 MNRAS 196, 111.

Sandage A., Saha A., Tammann, Pangia, Macchetto 1992, Science with the HST, ECF conference June 29 - July 7, 1992, Sardinia, in press.

Solf J. 1992 A\&A 257, 228.

Tuchman Y. 1991 ApJ 383, 779.

Weinberg M.D. 1992a ApJ 384, 81.

Weinberg M.D. 1992b ApJ 392, L67.

Whitelock P.A. 1990 in Confrontation between Stellar Pulsation and Evolution, eds. C. Cacciari and G. Clementini (ASP Conf. Ser. 11), p 365.

Whitelock P.A., Feast M., Catchpole R. 1991 MNRAS 248, 276. 
Whitelock P.A., Catchpole R. 1992 in The Centre, Bulge and Disk of the Milky Way, ed. L.Blitz (Kluwer: Dordrecht), in press.

Willems F.J., de Jong T. 1988 A\&A 196, 173.

Willson L.A. 1982 in Pulsations in Classical and Cataclysmic Variable Stars, eds. J.P.Cox and C.J.Hansen (Boulder: JILA), p 269.

Wood P.R. 1990a in From Miras to PN: Which Path for Stellar Evolution?, eds. M.O. Mennessier and A. Omont (Gif sur Yvette: Editions Frontières), p 67.

Wood P.R. 1990 b in Confrontation between Stellar Pulsation and Evolution, eds. C. Cacciari and G. Clementini (ASP Conf. Ser. 11), p 355.

Wood P.R., Bessell M.S. 1985 PASP 97, 681.

Wood P.R., Bessell M.S., Fox M.W. 1983 ApJ 272, 99.

Wood P.R., Hughes S.M.G. 1992, in preparation.

Wood P.R., Moore G.K.G., Hughes S.M.G. 1991 in IAU Symp. 148. The Magellanic Clouds, eds. R. Haynes and D. Milne (Kluwer: Dordrecht), p259.

Wood P.R., Whiteoak J.B., Hughes S.M.G., Bessell M.S., Gardner F.F., Hyland A.R. 1992, ApJ in press (October).

\section{Questions:}

D. Welch: Robertson (1974) found a number of RSGs in MC clusters. Have there been any additional cluster searches?

Hughes: The $I$ amplitudes of RSG LPVs are generally too low to have been detected in the UK Schmidt $I$ surveys. However, there are at least two groups (Mateo et al and Wood et al, private communications) that are currently monitoring several $\mathrm{MC}$ clusters with CCD photometry, and these are finding RSG LPVs.

H. Shibahashi: Can the mass and radius of individual stars be determined by comparing the theoretical evolutionary tracks and the observations on the $P L$ diagram?

Hughes: Pulsation masses were derived by Wood, Bessell and Fox (1983) for the MC LPVs, and more recent theoretical pulsation formulae that take into account metallicity effects are given in Wood $(1992 \mathrm{a}, 1992 \mathrm{~b})$. Radii can be calculated from the luminosities and temperatures, but would be very uncertain due to the still poorly determined temperatures for these very late-type stars. 